\title{
Simulação de bombas com velocidade de rotação variável no EPANET
}

\author{
Variable-speed pumps simulation in EPANET
}

Ricardo Santos Coutinho', Alexandre Kepler Soares ${ }^{2}$

$\square$

\begin{abstract}
RESUMO
Em sistemas de distribuição de água, economia de energia elétrica pode ser alcançada pela substituição de bombas de rotação constante por bombas de rotação variável (BRV). Os elevados custos de instalação e operação de BRV requerem uma avaliação econômica prévia do projeto, a qual geralmente é acompanhada do uso de simuladores para análises hidráulicas do sistema. Um dos programas mais utilizados para esse fim é o EPANET 2, que é impreciso para computar a eficiência de BRV, pois o programa não leva em conta a mudança na curva de eficiência da bomba quando sua velocidade de rotação é alterada. Neste trabalho, uma rotina para o cálculo da eficiência de BRV, baseada em metodologia da literatura, foi implementada no código-fonte do EPANET 2. A influência da nova rotina nos resultados previstos pelo programa para eficiência, potência e consumo de energia de BRV foi avaliada em simulações com uma rede hipotética. Os resultados mostram que a versão original do EPANET 2 tende a subestimar a eficiência e, consequentemente, superestimar a potência e o consumo de energia de BRV. A versão modificada do EPANET 2 proporciona uma melhor previsão do comportamento de BRV e, portanto, o seu uso é recomendado na simulação de redes de distribuição de água equipadas com BRV.
\end{abstract}

Palavras-chave: eficiência; bomba de rotação variável; rede de distribuição deágua.

\begin{abstract}
In water distribution systems, energy savings can be achieved by substituting fixed-speed pumps by variable-speed pumps (VSP). The high costs of installation and operation of VSP require a preliminary economic assessment of the project, which is usually complemented by the use of a computer simulation software for hydraulic analyses of the system. One of the most used software programs for this purpose is EPANET 2, which is inaccurate when computing VSP efficiency. This is because the software does not take into account the changes in the pump efficiency curve when the pump speed is changed. In this study, a routine for calculating VSP efficiency, based on literature methodology, was implemented in the source code of EPANET 2. The influence of the new routine on the results predicted by the software for efficiency, power and energy consumption of VSP was evaluated in simulations with a hypothetical network. The results show that the original version of EPANET 2 tends to underestimate the efficiency and thus overestimate the power and energy consumption of VSP. The modified version of EPANET 2 provides a better prediction of VSP behavior; thus, its use is recommended in the simulation of water distribution networks equipped with VSP.
\end{abstract}

Keywords: efficiency; variable-speed pump; water distribution network. $\square$

\section{INTRODUÇÃO}

A despesa com energia elétrica está entre os principais custos operacionais da atividade de abastecimento de água. Estima-se que esse item corresponda a entre 5 e $30 \%$ dos custos operacionais totais dos prestadores de serviços de água e esgoto, podendo chegar a $40 \%$ em países subdesenvolvidos ou em desenvolvimento. Em sistemas de distribuição de água, a maior parte da energia elétrica é consumida no bombeamento (CARLSON \& WALBURGER, 2007; IBARRA \& ARNAL, 2014). Assim, uma melhoria, ainda que pequena, na eficiência operacional das bombas pode resultar em uma economia significativa de energia e de custos para as companhias de água (GIUSTOLISI et al., 2013). Nesse sentido, deve-se considerar a possibilidade de substituição de

bombas com velocidade de rotação constante por bombas com velocidade de rotação variável (BRV).

Quando comparadas às bombas de rotação constantes, as BRV têm a vantagem de adaptar-se de forma mais eficiente às exigências do sistema, permitindo manter a pressão e a vazão próximas dos níveis mínimos exigidos, o que representa um potencial de economia de energia (WU et al., 2012). Todavia, os altos custos associados a implantação e operação dessa tecnologia requerem uma análise prévia de viabilidade econômica do projeto. A estimativa da economia de energia que pode ser alcançada com sistemas de BRV geralmente é acompanhada do uso de pacotes computacionais para simular o comportamento da rede de distribuição de água em que se pretende implantar tais sistemas.

\section{$\square$}

'Mestre em Engenharia do Meio Ambiente pela Universidade Federal de Goiás (UFG). Analista Ambiental, Engenheiro Sanitarista do Ministério Público do Estado de Goiás (MPGO) - Goiânia (GO), Brasil.

${ }^{2}$ Doutor em Engenharia Hidráulica e Saneamento pela Escola de Engenharia de São Carlos da Universidade de São Paulo (USP). Professor Associado da UFG - Goiânia (GO), Brasil. Endereço para correspondência: Alexandre Kepler Soares - Avenida Universitária, 1488 - Setor Universitário - 74605-220 - Goiânia (GO), Brasil - E-mail: aksoares@gmail.com Recebido em: 04/05/16 - Aceito em: 15/08/16 - Reg. ABES: 163428 
Entre os pacotes computacionais disponíveis para tal fim destaca-se o simulador hidráulico EPANET 2 (ROSSMAN, 2000a). Desenvolvido pela United States Environmental Protection Agency (EPA) e de distribuição gratuita, o EPANET 2 é amplamente utilizado para simular o comportamento de redes de água em regime permanente, por contar com um algoritmo de análise hidráulica extremamente eficiente denominado "método gradiente" (TODINI \& PILATI, 1988). Todavia, o programa apresenta uma imprecisão no cômputo da eficiência de $\mathrm{BRV}$, o que resulta em estimativas errôneas da potência das bombas e do consumo de energia do sistema de distribuição de água.

Assim, o presente trabalho teve o objetivo de implementar uma rotina para a correção da eficiência de BRV no EPANET 2 e avaliar sua influência nos resultados previstos pelo programa para eficiência, potência e energia consumida pelas bombas de uma rede de distribuição de água.

\section{BOMBAS DE ROTAÇÃO VARIÁVEL}

Bombas de rotação variável são bombas com motores acoplados a inversores de frequência, equipamentos capazes de alterar a frequência do sinal elétrico para que as bombas possam operar em diferentes velocidades de rotação. Quando comparadas às bombas de rotação constantes, as BRV têm a vantagem de adaptarem-se de forma mais eficiente às exigências do sistema, possibilitando uma melhor resposta às situações de anormalidade, como incêndios e rompimentos de tubulações (WOOD \& REEDY, 1995). Além disso, com o emprego dessa tecnologia, podem-se manter as pressões e as vazões perto dos níveis mínimos permitidos, o que representa um potencial de economia de energia (WU et al., 2012).
A operação da bomba em diferentes velocidades de rotação resulta em alterações em suas curvas características e, consequentemente, em seu desempenho. Tais alterações no comportamento da bomba podem ser previstas pelas relações de semelhança. Parte-se do pressuposto de que bombas geometricamente semelhantes trabalham em condições de semelhança, desde que tenham a mesma eficiência, ou seja, as características adimensionais da bomba permanecem constantes.

Para o caso particular em que somente a velocidade de rotação é modificada, as Equações 1, 2 e 3, conhecidas como relações de semelhança, podem ser derivadas:

$\frac{\mathrm{Q}_{1}}{\mathrm{Q}_{2}}=\frac{\mathrm{N}_{1}}{\mathrm{~N}_{2}}$

$\frac{\mathrm{H}_{1}}{\mathrm{H}_{2}}=\left(\frac{\mathrm{N}_{1}}{\mathrm{~N}_{2}}\right)^{2}$

$\frac{\mathrm{P}_{1}}{\mathrm{P}_{2}}=\left(\frac{\mathrm{N}_{1}}{\mathrm{~N}_{2}}\right)^{3}$

Onde:

Q: vazão da bomba $\left(\mathrm{m}^{3} / \mathrm{s}\right)$;

$\mathrm{H}$ : altura manométrica da bomba $(\mathrm{m})$;

$\mathrm{P}$ : potência da bomba $(\mathrm{W})$;

$\mathrm{N}$ : velocidade de rotação da bomba (rpm);

1 e 2: duas velocidades distintas de rotação.

A Figura 1 mostra que a curva eficiência $(\eta)$ versus coeficiente adimensional de vazão $\left(\mathrm{C}_{\mathrm{Q}}\right)$ não é alterada com a mudança na velocidade

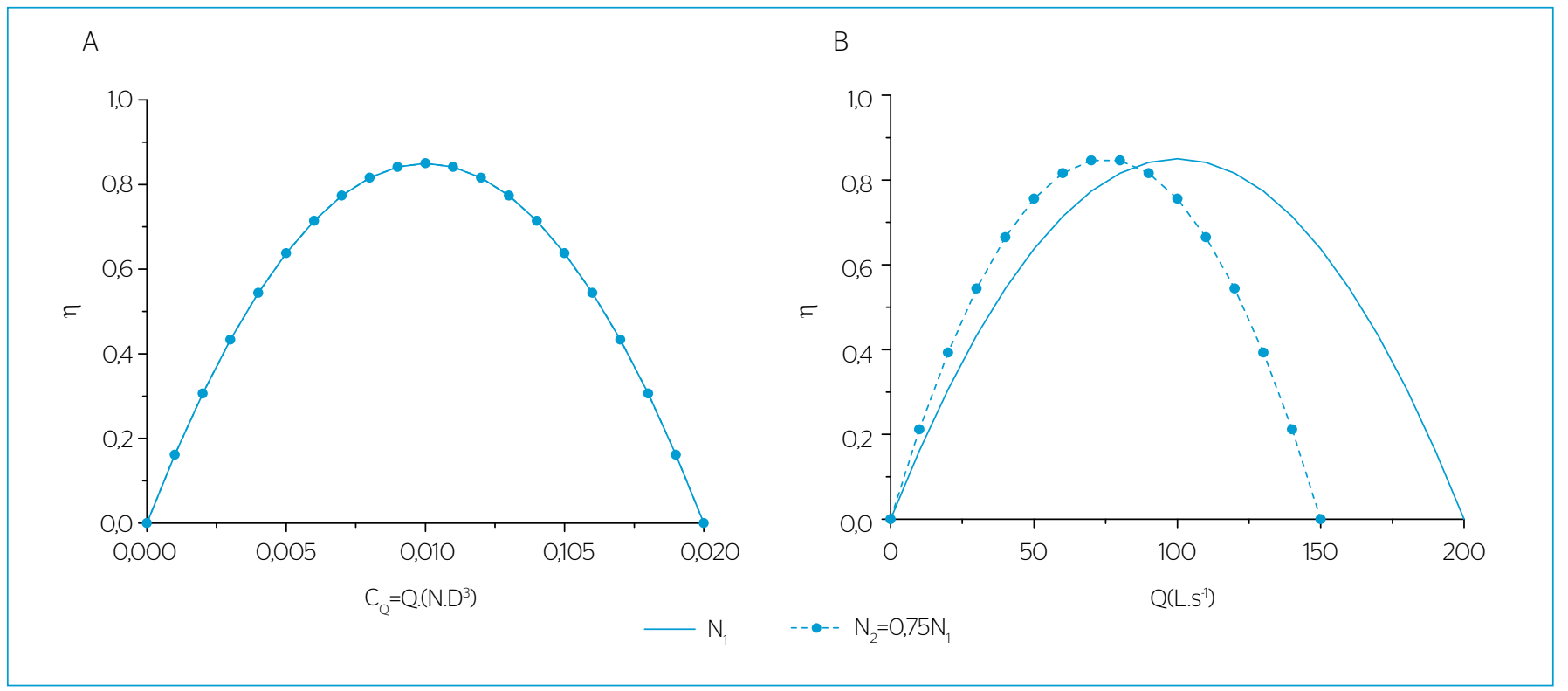

Figura 1 - Curvas de eficiência em duas velocidades de rotação; (A) $\eta \times C_{Q}$; (B) $\eta \times Q$. 
de rotação da bomba. O mesmo não ocorre com a curva eficiência ( $\eta$ ) versus vazão (Q), que é redimensionada segundo a Equação 1.

Simpson e Marchi (2013) apontam que as relações de semelhança não levam em conta os fatores que não se ajustam com a velocidade e cuja magnitude depende do tamanho da máquina, o que pode resultar em estimativas com erros relativos significativos, em especial para a eficiência de bombas de pequeno porte. Ao comparar a eficiência prevista pelas relações de semelhança com a medida pelo fabricante para o ponto de melhor eficiência de três bombas de diferentes portes (556, 90 e 5,5 kW), os autores verificaram que uma redução de 22,5 e $44,4 \%$ na velocidade de rotação das bombas de maior e menor porte, respectivamente, resultou em erros relativos de 0,12 e 7,69\% entre as eficiências estimadas e medidas, respectivamente.

A diminuição da eficiência quando a velocidade de rotação é reduzida foi reportada por vários autores. Morton (1975) propôs um método gráfico para identificar a nova curva, enquanto Sárbu e Borza (1998) relacionaram a nova eficiência com a eficiência original e com a velocidade de rotação da bomba. Abordagens mais generalistas consideraram que a eficiência é uma função do número de Reynolds. Gulich (2003) aponta que um dos primeiros métodos para ajustar a eficiência de bombas semelhantes foi formulado observando que somente uma parte das perdas de energia é função do número de Reynolds, conforme a Equação 4:

$\frac{\left(1-\eta_{2}\right)}{\left(1-\eta_{1}\right)}=K+(1-K)\left(\frac{\operatorname{Re}_{1}}{\operatorname{Re}_{2}}\right)^{\mathrm{m}}$

Onde:

Re: número de Reynolds;

$\eta$ : eficiência;

K: perdas;

1 e 2: duas velocidades distintas de rotação;

$\mathrm{m}$ : expoente de relação entre os números de Reynolds referentes a duas velocidades distintas de rotação.

Assumindo que todas as perdas de energia são independentes do número de Reynolds, ou seja, $K=0$, e que o valor de $m$ é igual a 0,1 , a Equação 4 pode ser reescrita como a fórmula de Sárbu e Borza (1998) (Equação 5). A razão entre os número de Reynolds $\left(\mathrm{Re}_{1} / \mathrm{Re}_{2}\right)$ pode ser substituída pela razão entre as velocidades de rotação da bomba $\left(\mathrm{N}_{1} / \mathrm{N}_{2}\right)$, tendo em vista que a velocidade do fluido é proporcional à velocidade de rotação da bomba, desde que o diâmetro e a viscosidade não mudem.

Embora a abordagem apresentada nas Equações 4 e 5 seja limitada pelo fato do expoente $\mathrm{m}$ depender do número de Reynolds e da rugosidade (OSTERWALDER, 1978; GULICH, 2003), a fórmula de Sárbu e Borza (1998) oferece bons resultados se a velocidade de rotação da bomba não for reduzida para menos de $70 \%$ da velocidade nominal (SIMPSON \& MARCHI, 2013):
$\eta_{2}=1-\left(1-\eta_{1}\right)\left(\frac{N_{1}}{N_{2}}\right)^{0,1}$

Onde:

$\eta$ : eficiência;

$\mathrm{N}$ : velocidade de rotação.

Simpson e Marchi (2013) utilizaram as relações de semelhança e a fórmula de Sárbu e Borza (1998) para estimar a eficiência do novo ponto de melhor eficiência de 3 bombas com potências iguais a 556,0; 90,0 e 5,5 kW e velocidades de rotação reduzidas para 77,5; 77,5 e $55,6 \%$ das respectivas velocidades de rotação nominal, sendo os resultados comparados às eficiências medidas pelos fabricantes. Verificou-se que os erros relativos das eficiências previstas pela fórmula de Sárbu e Borza (1998) foram inferiores aos das eficiências previstas pelas relações de semelhança para as bombas de pequeno e de médio porte, e superior a essas para a bomba de grande porte. Diante desses resultados, os autores concluíram que, apesar de ainda representar uma aproximação, a fórmula de Sárbu e Borza (1998) permite uma estimativa mais acurada da eficiência para uma ampla faixa de velocidades de rotação quando comparada às relações de semelhança e recomendam o uso dessa fórmula para diminuir o erro na estimativa da eficiência, especialmente para bombas de pequeno porte.

O EPANET 2 utiliza as relações de semelhança dadas pelas Equações 1 e 2 para estimar a vazão e a altura manométrica da bomba quando essa tem sua velocidade de rotação alterada. Todavia, não utiliza a Equação 3 para calcular a potência. Em vez disso, o programa usa a curva eficiência versus vazão na velocidade nominal de rotação, que geralmente é fornecida pelo fabricante.

Conforme mencionado, a mudança na velocidade de rotação da bomba provoca alterações em suas curvas características. No caso da curva eficiência versus vazão, a redução da velocidade de rotação da bomba causa um estreitamento e deslocamento à esquerda da curva, mas mantém o mesmo ponto de melhor eficiência (Figura 1). O estreitamento da curva ocorre porque o intervalo de vazões sobre o qual a bomba pode operar é reduzido na mesma proporção que a velocidade de rotação, conforme Equação 1.

Ao simular o comportamento de BRV, o EPANET 2 modela corretamente as mudanças na curva $\mathrm{H}$ x Q. Todavia, o programa continua a utilizar a curva $\eta \times \mathrm{Q}$ na velocidade nominal de rotação e, desse modo, falha em identificar a eficiência do novo ponto de operação da bomba. Marchi e Simpson (2013) compararam a potência estimada pelo EPANET 2 com a prevista pelas relações de semelhança. Para isso, utilizaram uma adutora hipotética, em que uma bomba é utilizada para recalcar a água de um reservatório de nível fixo para um reservatório de nível variável. A velocidade de rotação da bomba foi reduzida para $76,5 \%$ da velocidade 
nominal de rotação $\left(\mathrm{N}_{2}=0,765 \mathrm{~N}_{1}\right)$, o que resultou na mudança de seu ponto de operação de A $\left(124,1{\mathrm{~L} . \mathrm{s}^{-1}}^{1} ; 120,8 \mathrm{~m}\right)$ para B (95 L.s ${ }^{-1}$; $70,8 \mathrm{~m})$, na curva H x Q, e de C (124,1 L.s $\left.{ }^{-1} ; 84,2 \%\right)$ para D (95 L.s ${ }^{-1}$; $84,2 \%)$, na curva $\eta \times \mathrm{Q}$, conforme mostra a Figura 2. Todavia, em vez de considerar a mudança de $\mathrm{C}$ para $\mathrm{D}$, o programa computou a mudança de C para E (95 L.s ${ }^{-1} ; 76,3 \%$ ). A potência correspondente ao ponto $\mathrm{D}$ foi de $78,3 \mathrm{~kW}$, enquanto a correspondente ao ponto $\mathrm{E}$ foi de $86,4 \mathrm{~kW}$, ou seja, o EPANET 2 superestimou a potência da bomba prevendo um valor $10,3 \%$ maior do que o obtido pelas relações de semelhança. Os autores chamam a atenção, também, para a importância de se incluir na avaliação da eficiência de BRV todos os componentes do conjunto elevatório, o que inclui motores e inversores de frequência, os quais atualmente não são modelados pelo EPANET 2.

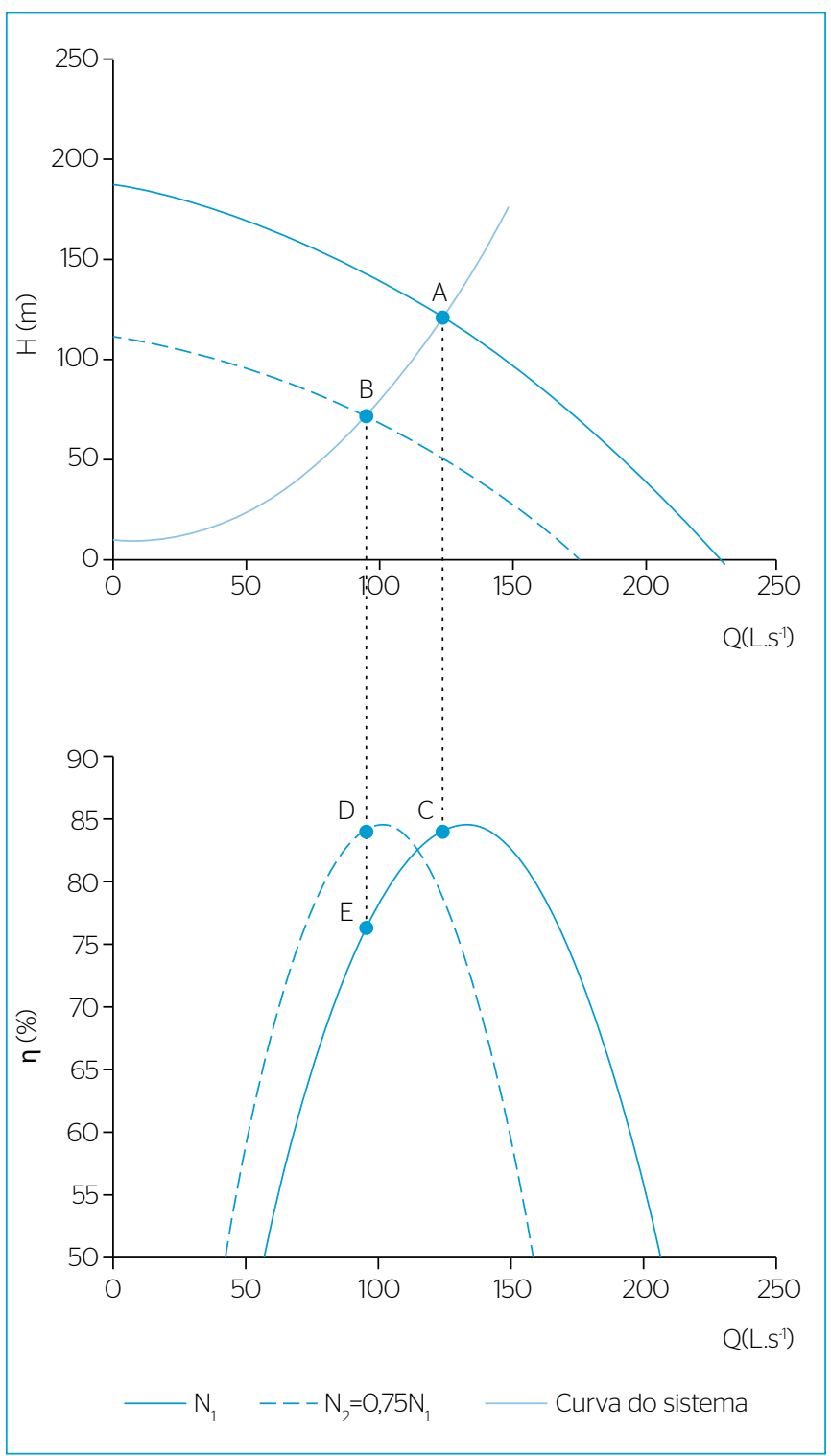

Figura 2 - Ponto de operação da bomba em duas velocidades de rotação.

\section{METODOLOGIA}

O pacote do simulador hidráulico EPANET 2 conta com uma extensão para desenvolvedores denominada EPANET 2 Programmer's Toolkit, a qual possui uma série de funções compiladas numa biblioteca de vínculo dinâmico (dynamic-link library - DLL), um arquivo de bibliotecas compartilhadas utilizadas em sistemas operacionais Microsoft Windows. A ferramenta permite a desenvolvedores personalizar os algoritmos de análise hidráulica e de qualidade da água implementados no EPANET de acordo com suas necessidades, podendo ser incorporada a aplicativos Windows 32-bit escritos em C/C++, Delphi Pascal, Visual Basic ou qualquer outra linguagem que seja capaz de chamar funções de um arquivo DLL (ROSSMAN, 2000b).

O EPANET 2 versão Toolkit foi codificado no padrão ANSI C com módulos separados de código para o processamento dos dados de entrada, análise hidráulica, análise da qualidade da água, análise de matrizes esparsas e equações lineares, e geração de relatórios. Todavia, como o módulo de análise hidráulica da Toolkit é o mesmo do código-fonte do EPANET 2, a ferramenta também é imprecisa no cálculo da eficiência de BRV. Para corrigir essa imprecisão da Toolkit, é necessário criar uma nova DLL a partir do código-fonte do EPANET 2. Obviamente, deve-se realizar as alterações necessárias no código-fonte do programa antes de se criar a nova DLL.

Assim, foram realizadas intervenções no código-fonte do EPANET 2 com duas finalidades:

1. calcular a eficiência de BRV; e

2. recuperar a eficiência das bombas como dado de saída da Toolkit.

Para isso, implementou-se no código-fonte do programa a fórmula de Sárbu e Borza (1998) para se estimar a eficiência das bombas e as modificações propostas por Marchi e Simpson (2013) para recuperar diretamente a eficiência das bombas. Os trechos dos códigos para tais modificações são propostos por Marchi e Simpson (2013) e são comentados por Coutinho (2015).

Após a implementação das alterações no código-fonte do EPANET 2, utilizou-se o compilador Microsoft Visual C++ 2010 Express para criar a nova DLL. Para tanto, adotou-se o procedimento descrito no Quadro 1. Ao final do processo, foram obtidos os arquivos epanet2.dll e epanet2.lib, que, em conjunto com o arquivo epanet2.h, são necessários para se compilar, em ambiente Microsoft Visual C++ 2010 Express, rotinas que busquem as funções do EPANET 2 definidas na DLL. Um procedimento alternativo para a criação da DLL, utilizando-se o compilador Borland C++ Builder 6, é apresentado por Coutinho (2015).

\section{Estudo de caso}

Neste trabalho, utilizou-se a rede hipotética Anytown (Walski et al., 1987) (Figura 3), com as alterações incluídas por Kurek e Ostfeld (2014) nas simulações hidráulicas realizadas no EPANET 2, versões original e modificada. A opção pela rede Anytown alterada por Kurek 
e Ostfeld (2014) deve-se ao fato do trabalho desses autores apresentar padrões diários de variação da velocidade de rotação das bombas, os quais foram obtidos após a otimização multiobjetivo da operação da referida rede. Conforme mostra a Figura 3, a rede em estudo consiste em dois mananciais que fornecem água, com auxílio de duas bombas, a uma rede de distribuição com 16 nós, 34 tubos e 1 reservatório de nível variável. As duas bombas do sistema operam sob rotação variável e possuem curvas características distintas. Os padrões de operação das bombas foram extraídos do trabalho de Kurek e Ostfeld (2014) e são apresentados na Figura 4, em que $\mathrm{N}$ representa a velocidade relativa de rotação da bomba, sendo definida como o quociente entre determinada velocidade de rotação $\mathrm{N}_{2}$ e a velocidade nominal de rotação $\mathrm{N}_{1}$.

A curva característica altura manométrica $(\mathrm{H})$ versus vazão $(\mathrm{Q})$ das bombas é gerada automaticamente pelo EPANET 2 a partir da informação de três pontos de operação para cada bomba: o primeiro para condição de vazão nula (ponto de shutoff); o segundo para a condição desejada

Quadro 1 - Procedimento para geração de nova dynamic-link library para o EPANET 2.

Com o auxílio de um editor de texto, abrir o arquivo epanet.c e habilitar a linha de definição da DLL destacada em negrito no trecho de código:

/*** New compile directives ***///(2.00.11 - LR)

//\#define CLE /* Compile as a command line executable */

//\#define SOL /* Compile as a shared object library */

\#define DLL /* Compile as a Windows DLL *

No prompt de comando do Microsoft Visual C++ 2010, acessar o diretório do código-fonte do EPANET 2 e digitar o comando:

cl -o epanet2.dll epanet.c hash.c hydraul.c inpfile.c input1.c input2.c

input3.c mempool.c output.c quality.c report.c rules.c smatrix.c /link /DLL de operação; e o terceiro para a condição de máxima vazão. O programa ajusta uma função potência aos dados disponíveis, conforme Equação 6 . A Figura 5 mostra as curvas $\mathrm{H} x \mathrm{Q}$ ajustadas pelo EPANET 2 aos pontos de operação inseridos como dados de entrada das bombas do sistema.

$\mathrm{H}=\mathrm{H}_{0}-\mathrm{RQ}^{\mathrm{n}}$

Onde:

$\mathrm{H}$ : altura manométrica da bomba $(\mathrm{m})$;

$\mathrm{H}_{0}$ : altura manométrica no ponto de shutoff (vazão nula) (m);

Q: vazão da bomba $\left(\mathrm{m}^{3} / \mathrm{s}\right)$;

R e n: constantes da função de ajuste.

As curvas $\mathrm{H}$ x Q mostradas na Figura 5 correspondem à velocidade nominal de rotação das bombas. Se a velocidade de rotação é alterada, as respectivas curvas $\mathrm{H}$ x Q também são alteradas no EPANET, conforme relações de semelhança das Equações 1 e 2. A relação entre a altura manométrica e a vazão das bombas para determinada velocidade de rotação é representada pela Equação 7.

$\mathrm{H}=\mathrm{N}^{2}\left[\mathrm{H}_{0}-\mathrm{R}\left(\frac{\mathrm{Q}}{\mathrm{N}}\right)^{\mathrm{n}}\right]$

Onde:

$\mathrm{H}$ : altura manométrica da bomba $(\mathrm{m})$ na velocidade de rotação $\mathrm{N}_{2}$; $\mathrm{H}_{0}$ : altura manométrica no ponto de shutoff $(\mathrm{m})$ na velocidade nominal de rotação $\mathrm{N}_{1}$;

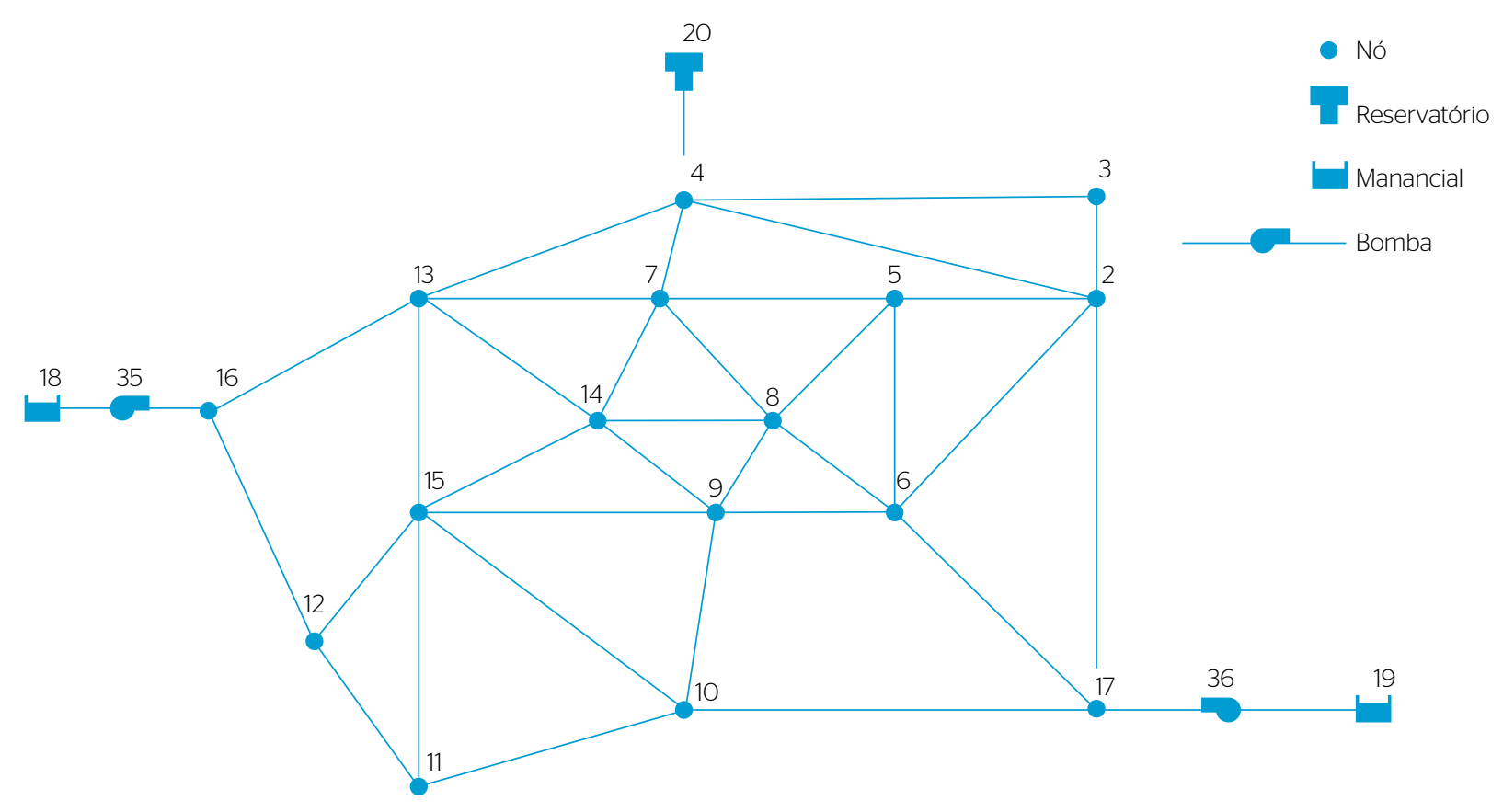

Figura 3 - Rede hipotética. 
Q: vazão da bomba $\left(\mathrm{m}^{3} / \mathrm{s}\right)$ na velocidade de rotação $\mathrm{N}_{2}$;

$\mathrm{R}$ e $\mathrm{n}$ : constantes da função de ajuste para a velocidade nominal de rotação $\mathrm{N}_{1}$;

$\mathrm{N}$ : velocidade relativa de rotação da bomba dada por $\mathrm{N}_{2} / \mathrm{N}_{1}$.

A curva eficiência versus vazão das bombas é definida por pontos representados por pares ordenados conhecidos, os quais são inseridos como dados de entrada das bombas. A partir desses dados, o EPANET 2 cria uma curva unindo os pontos com seguimentos de reta. Para as bombas da rede em estudo, arbitraram-se os pares ordenados (Q; $\eta)$ apresentados na Figura 6.

As curvas $\eta \times \mathrm{Q}$ apresentadas na Figura 6 referem-se à velocidade nominal de rotação das bombas. Se a velocidade é alterada, as respectivas curvas de eficiência também o são. Todavia, o EPANET 2 não leva em conta as mudanças na curva de eficiência decorrentes da alteração da velocidade de rotação da bomba e continua a utilizar as curvas $\eta \mathrm{x} \mathrm{Q}$ correspondentes à velocidade nominal de rotação para estimar a eficiência do novo ponto de operação das bombas. Assim, foram determinadas as curvas teóricas de eficiência para as velocidades relativas de rotação previstas no padrão operacional das bombas (Figura 4). Para isso, ajustou-se um polinômio de grau 2 aos pares ordenados $(\mathrm{Q} ; \eta)$ conhecidos para a velocidade nominal de rotação e aplicou-se a ele a relação de semelhança dada pela Equação 1, conforme sugerido por Sá Marques e Sousa (2007). Desse modo, a curva de eficiência correspondente a uma velocidade de rotação qualquer está representada pela Equação 8.
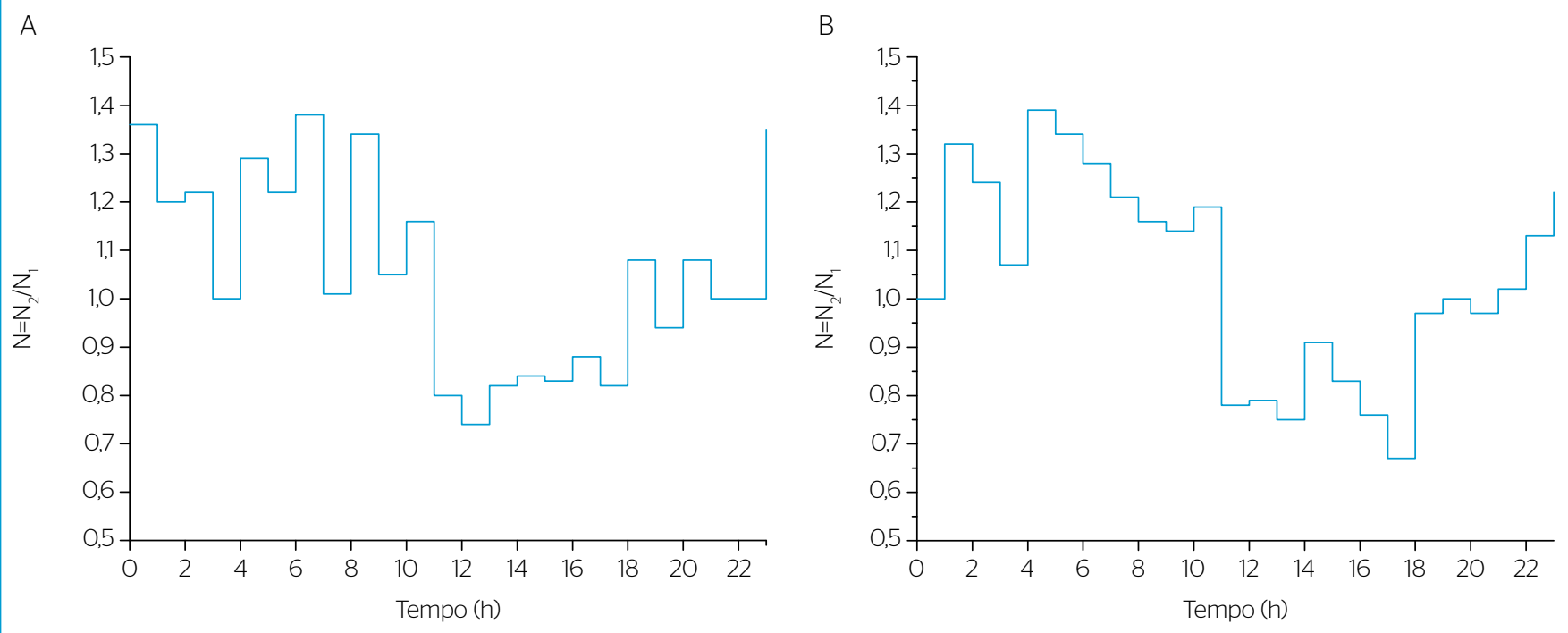

Figura 4 - Padrão de variação da velocidade de rotação; (A) bomba 35; e (B) bomba 36.

A

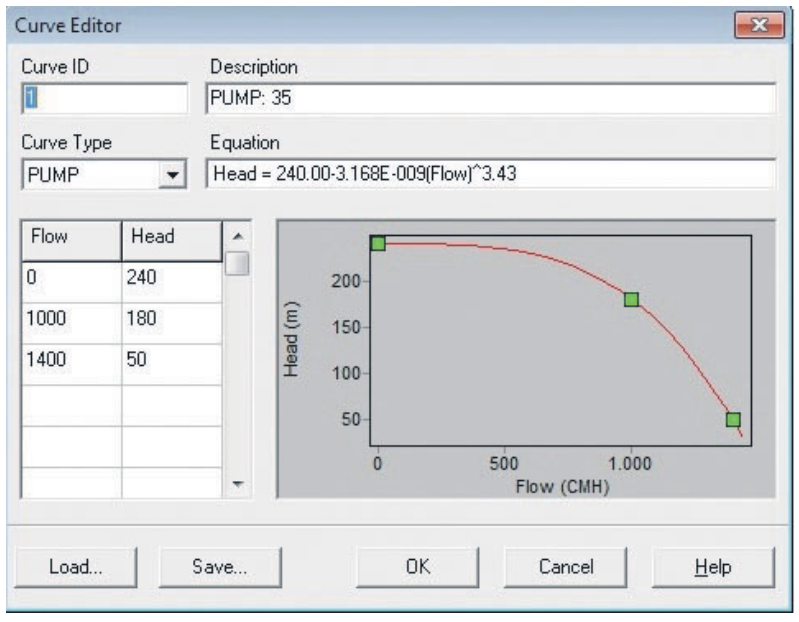

$\mathrm{B}$

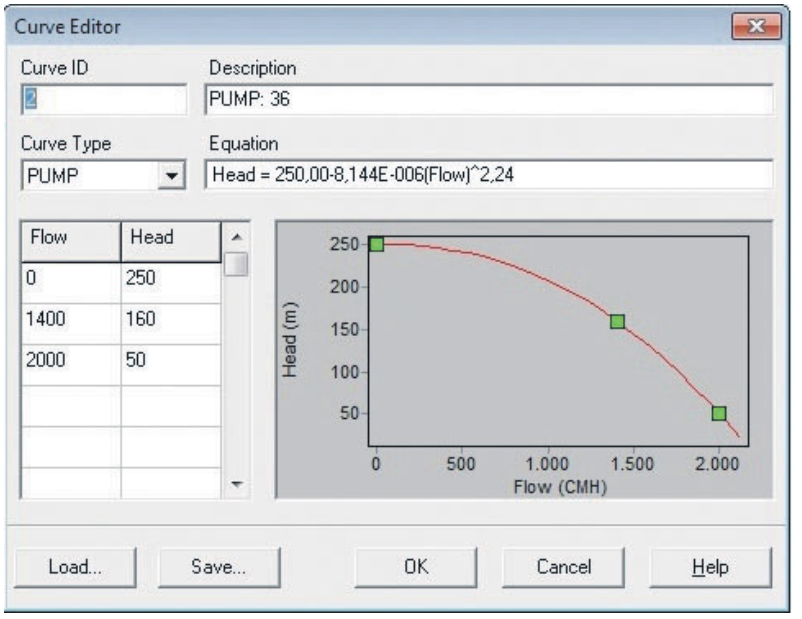

Figura 5 - Curvas H x Q; (A) bomba 35; e (B) bomba 36. 
$\eta=A\left(\frac{Q}{N}\right)^{2}+B\left(\frac{Q}{N}\right)+C$

Onde:

$\eta$ : eficiência da bomba na velocidade de rotação $\mathrm{N}_{2}$;

Q: vazão da bomba na velocidade de rotação $\mathrm{N}_{2}$;

$\mathrm{N}$ : velocidade relativa de rotação $\left(\mathrm{N}_{2} / \mathrm{N}_{1}\right)$;

A, B, C: constantes da função de ajuste para a velocidade nominal de rotação $\mathrm{N}_{1}$.

\section{RESULTADOS}

As simulações realizadas com a rede Anytown para condições de bombeamento com eficiência variável tiveram por finalidade avaliar a influência das intervenções feitas no código-fonte do EPANET 2 na previsão da eficiência e, consequentemente, da potência e do consumo de energia de BRV. Para isso, foi necessário informar, como dado de entrada do modelo, as curvas de eficiência e padrões de variação da velocidade de rotação das bombas.

Os resultados de eficiência previstos pelo EPANET 2 original foram comparados aos valores teóricos obtidos pela fórmula de Sárbu e Borza (1998), a qual foi implementada na versão modificada do referido simulador. A utilização da fórmula de Sárbu e Borza (1998) para se estimar a eficiência $\eta_{2}$ do novo ponto de operação de uma bomba, quando esta tem sua velocidade de rotação alterada, requer apenas o conhecimento da eficiência $\eta_{1}$ do ponto homólogo na curva $\eta \times \mathrm{Q}$, correspondente à velocidade nominal de rotação, e da razão da velocidade de rotação atual $\mathrm{N}_{2}$ para a velocidade de rotação nominal $\mathrm{N}_{1}$, ou seja, $\mathrm{N}_{2} / \mathrm{N}_{1}$. Desse modo, e a partir da Equação 5, é possível determinar a eficiência teórica de $\eta_{2}$ para diferentes valores de $\eta_{1}$ e de $N_{2} / N_{1}$ (Figura 7). Observa-se, na Figura 7, que a eficiência estimada $\eta_{2}$ aproxima-se da eficiência original $\eta_{1}$ se essa é elevada e a variação na velocidade de rotação da bomba é pequena. Para valores de $\eta_{1}$ superiores a 0,70 e variações na velocidade de rotação da bomba inferiores a $30 \%\left(0,7<\mathrm{N}_{2} / \mathrm{N}_{1}<1,3\right)$, por exemplo, a diferença entre as eficiências $\eta_{2}$ e $\eta_{1}$ é inferior a 1,6\%. Todavia, a diferença entre $\eta_{2}$ e $\eta_{1}$ tende a aumentar quanto menor o valor dessa última e maior o valor de $\mathrm{N}_{2} / \mathrm{N}_{1}$.

A diferença entre as eficiências estimada e original é proporcionalmente maior em reduções do que em aumentos na velocidade de rotação da bomba. No caso de $\eta_{1}$ igual a 0,6 e variações de $\pm 30 \%$ na velocidade de rotação da bomba, por exemplo, a diferença entre as eficiências estimada e original é de $-2,42 \%$ para a redução e $1,73 \%$ para o aumento na velocidade de rotação da bomba, conforme mostra a Figura 8.

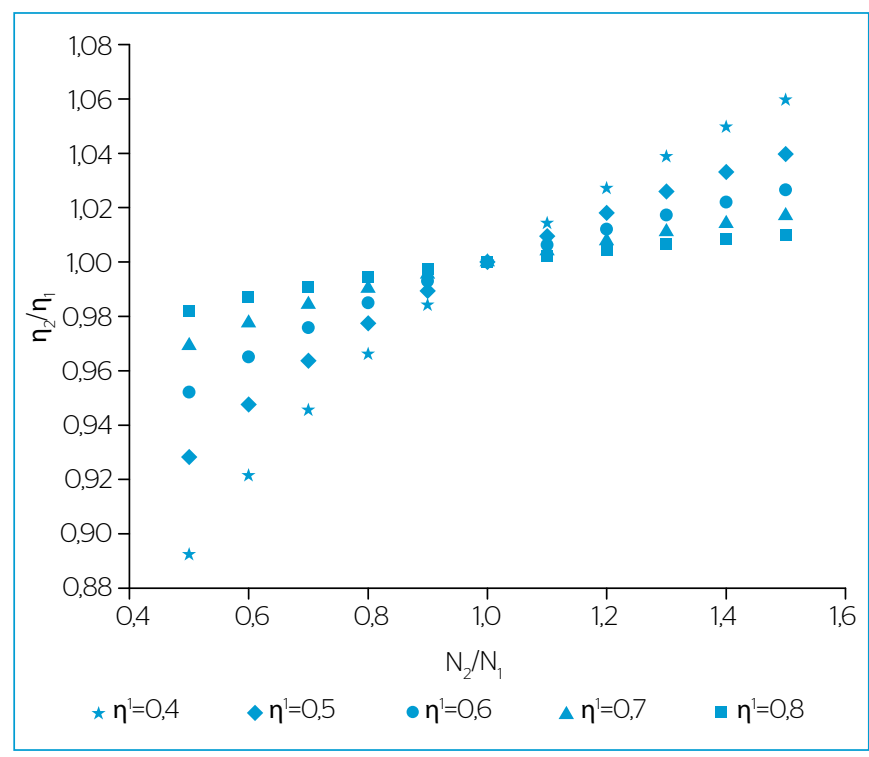

Figura 7 - Eficiências estimadas pela fórmula de Sárbu e Borza (1998).
A

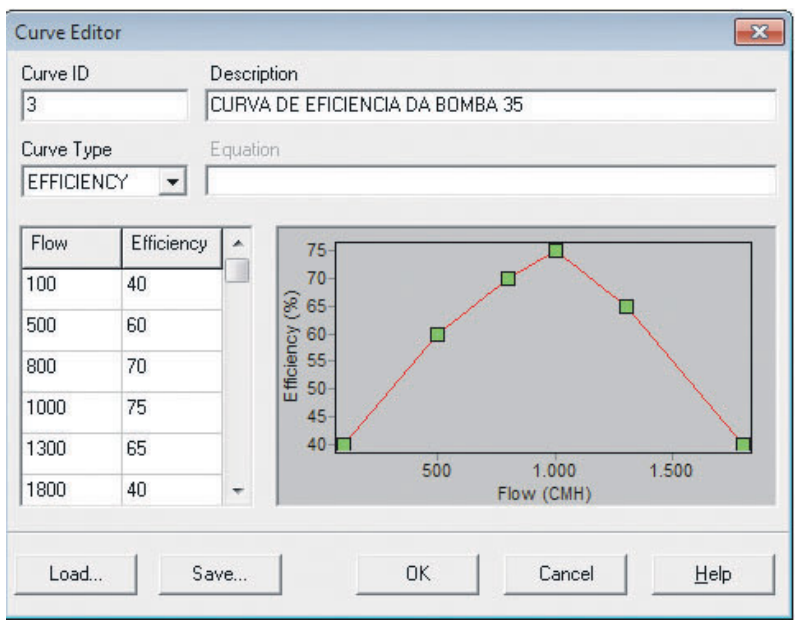

$\mathrm{B}$

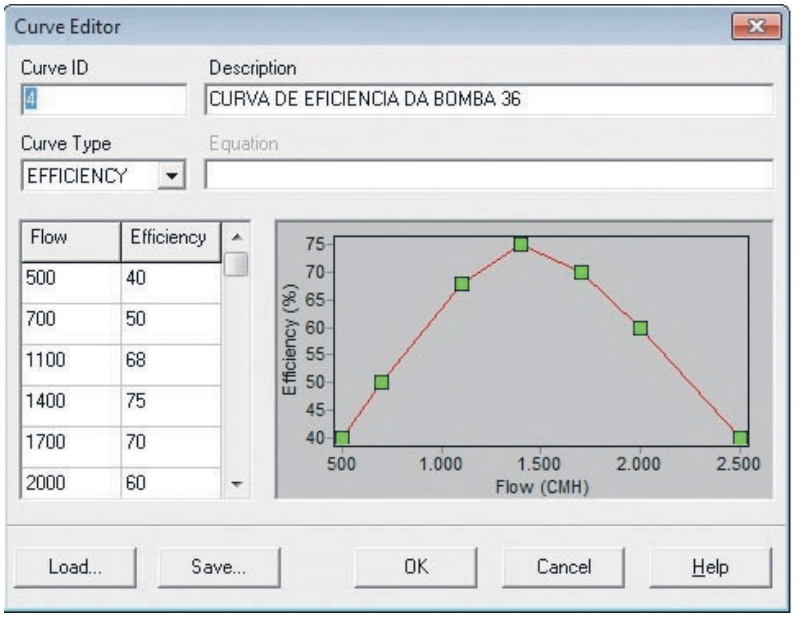

Figura 6 - Curvas h x Q; (A) bomba 35; e (B) bomba 36. 
No caso da rede Anytown, a eficiência das bombas em cada intervalo de tempo depende de suas curvas características e padrões de operação em termos de velocidade de rotação. A partir dessas informações é determinado o valor de $\eta_{1}$ com o auxílio das relações de semelhança (Equações 1 e 2) e, em seguida, o valor de $\eta_{2}$ pela fórmula de Sárbu e Borza (1998) (Equação 5).

A Figura 9 apresenta as eficiências horárias previstas pelo EPANET 2, versões original e modificada, para as bombas da rede em estudo. Observa-se que as eficiências previstas pelo EPANET 2 original foram diferentes das previstas pelo EPANET 2 modificado, nos intervalos de tempo em que houve mudança na velocidade de rotação das bombas.

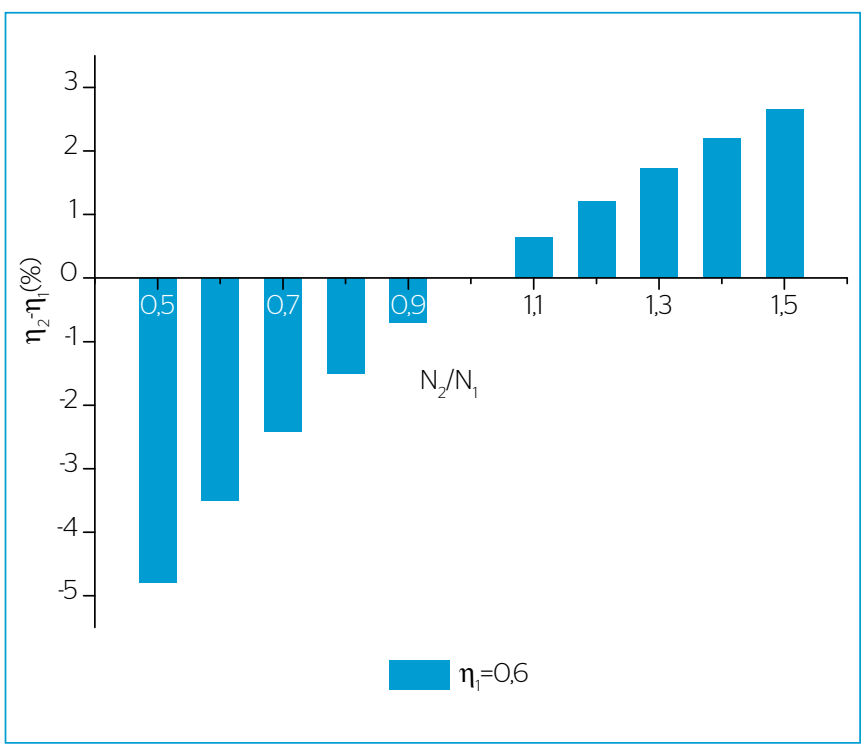

Figura 8 - Eficiências estimada $\left(\eta_{2}\right)$ e original $\left(\eta_{1}\right)$ para diferentes variações $\left(\mathrm{N}_{2} / \mathrm{N}_{1}\right)$.
Na maior parte do ciclo operacional, a versão original do simulador subestimou a eficiência das bombas.

A Figura 10 apresenta a variação do fator de correção $\eta_{2} / \eta_{1}$ para as bombas do sistema ao longo do ciclo operacional. Em vários intervalos de tempo os resultados de $\eta_{2} / \eta_{1}$ previstos pelo EPANET 2 original ou assumem valores superiores à unidade, para variações negativas na velocidade de rotação das bombas, ou inferiores à unidade, para variações positivas na velocidade de rotação das bombas - ao contrário do que prevê a fórmula de Sárbu e Borza (1998). Por exemplo, no tempo $19 \mathrm{~h}$ da bomba 35, foi previsto um fator de correção $\eta_{2} / \eta_{1}$ igual a 1,0212 , para uma redução de $6 \%$ na velocidade de rotação da bomba e eficiência original $\left(\eta_{1}\right)$ igual a 0,7321 , quando o resultado esperado era de 0,9978 . Já no tempo $4 \mathrm{~h}$ da bomba 36 , foi previsto um $\eta_{2} / \eta_{1}$ igual a 0,6320 , para um aumento de $39 \%$ na velocidade de rotação da bomba e eficiência original $\left(\eta_{1}\right)$ igual a 0,6880 , quando o resultado esperado era de 1,0147.

A Figura 11 ilustra duas curvas teóricas $\eta \times \mathrm{Q}$ e as eficiências previstas pelo EPANET 2, versões original e modificada, para dois intervalos de tempo do ciclo operacional. A diminuição na velocidade de rotação das bombas provoca estreitamento e deslocamento à esquerda da curva $\eta \times$ Q. Por outro lado, o aumento na velocidade de rotação das bombas provoca alargamento e deslocamento à direita da curva $\eta \times$ Q. Obviamente, a intensidade das alterações na curva de eficiência é tanto maior quanto maior a variação na velocidade de rotação da bomba.

A Figura 12 apresenta as potências estimadas pelo EPANET 2, versões original e modificada, para as bombas da rede em estudo, quando essas têm a velocidade de rotação alterada de acordo com

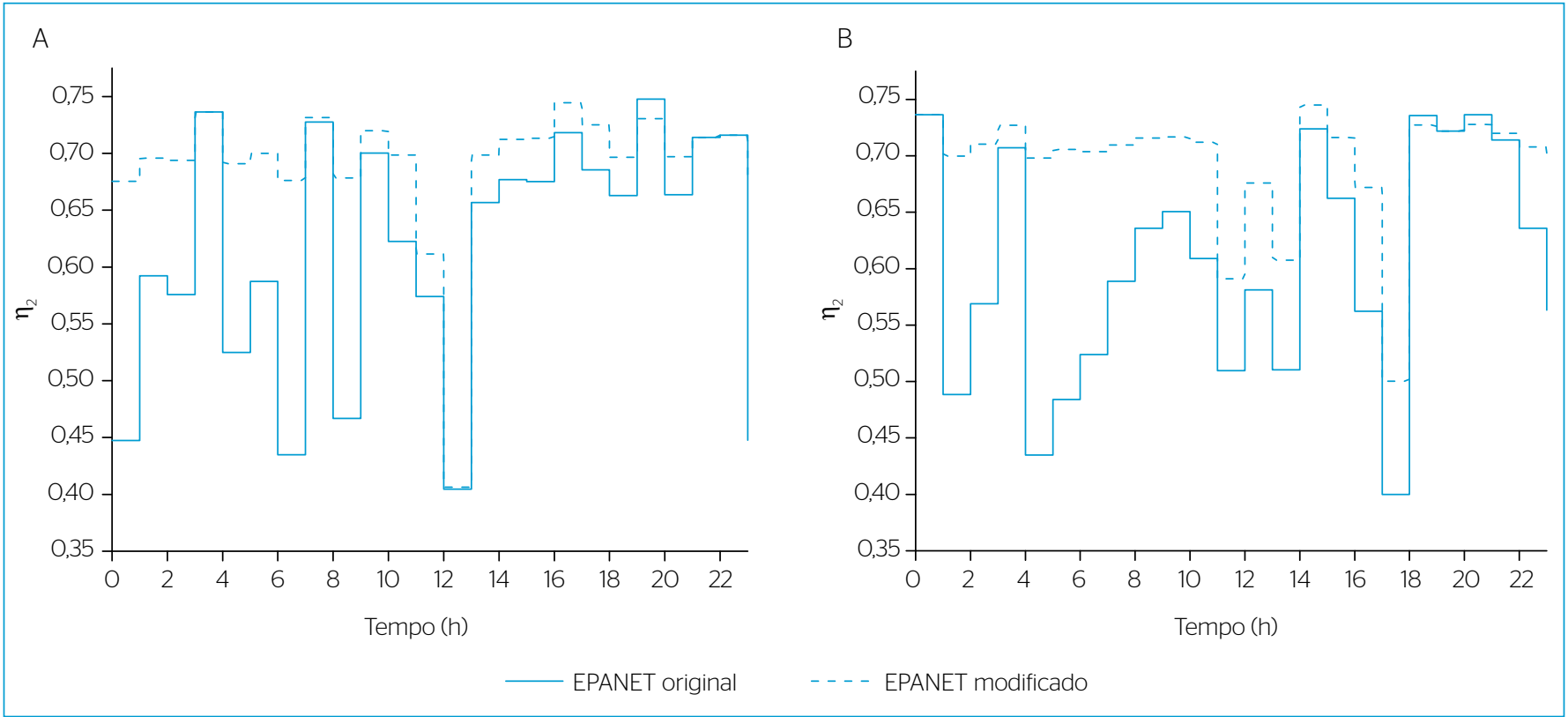

Figura 9 - Eficiências previstas pelo EPANET 2, versões original e modificada; (A) bomba 35 e (B) bomba 36. 
os padrões mostrados na Figura 4. Observa-se que as potências previstas pelo EPANET 2 original foram superiores às previstas pelo EPANET 2 modificado, para ambas as bombas e durante a maior parte do ciclo operacional. Houve, entretanto, intervalos de tempo em que as potências estimadas pelo simulador hidráulico original foram inferiores às previstas pelo simulador modificado. As potências previstas pelo EPANET 2 original variaram de $96,63 \mathrm{~kW}(\mathrm{t}=12 \mathrm{~h})$ a $2265,14 \mathrm{~kW}(\mathrm{t}=6 \mathrm{~h})$, para a bomba 35 , e de $347,51 \mathrm{~kW}(\mathrm{t}=17 \mathrm{~h}) \mathrm{a}$ $3046,00 \mathrm{~kW}(\mathrm{t}=4 \mathrm{~h})$, para a bomba 36. Já as eficiências estimadas pelo
EPANET 2 modificado variaram de $96,25 \mathrm{~kW}(\mathrm{t}=12 \mathrm{~h})$ a $1457,26 \mathrm{~kW}$ $(\mathrm{t}=6 \mathrm{~h})$, para a bomba 35 , e de $277,91 \mathrm{~kW}(\mathrm{t}=17 \mathrm{~h})$ a $1897,21 \mathrm{~kW}$ $(\mathrm{t}=4 \mathrm{~h})$, para a bomba 36 .

A Tabela 1 apresenta o erro relativo entre a potência prevista pelo EPANET 2 original e a prevista pelo EPANET 2 modificado, considerando o valor dessa última como o verdadeiro, para cada intervalo de tempo do ciclo operacional. Nota-se que o menor erro relativo entre as potências estimadas pelos simuladores hidráulicos considerados foi de $0,39 \%(t=12 \mathrm{~h})$, para a bomba 35 , e de

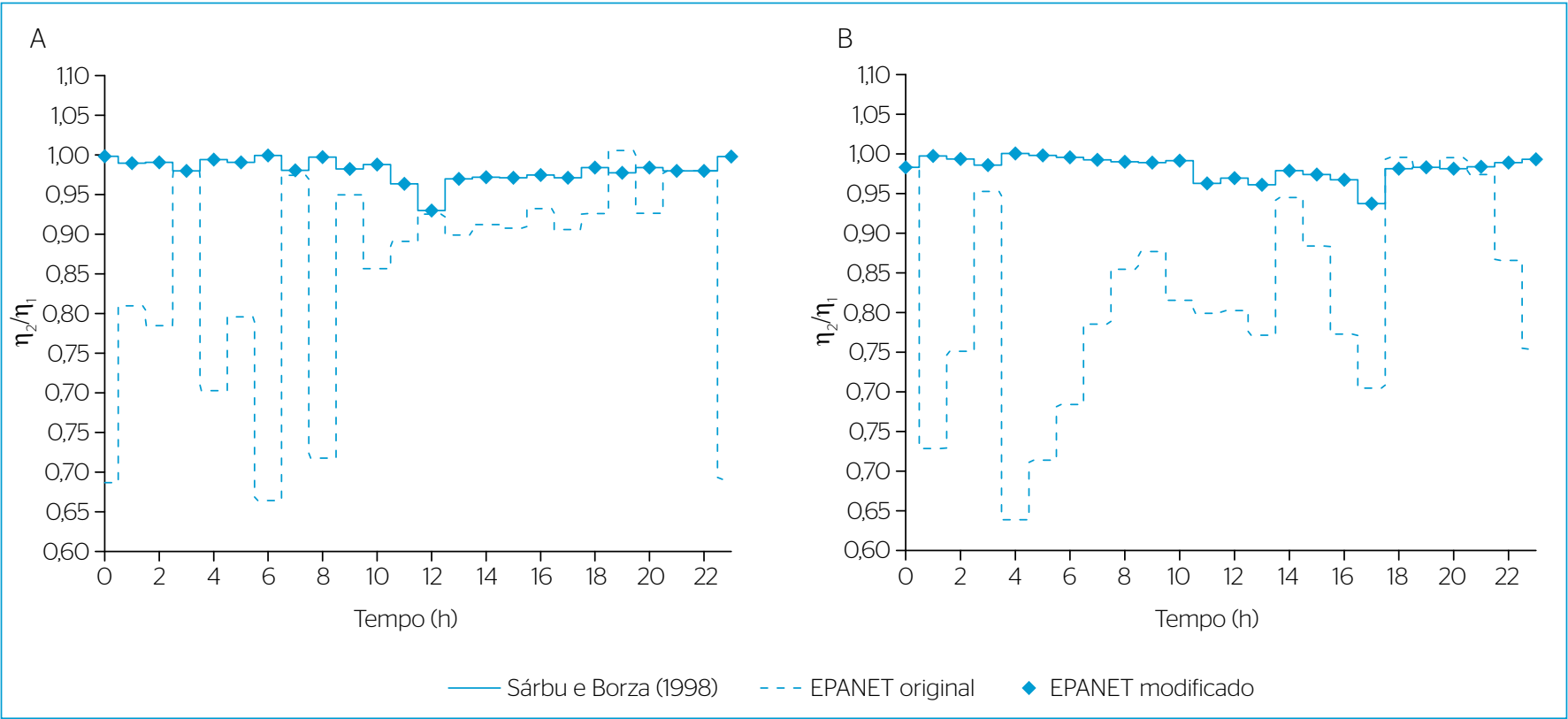

Figura 10 - Resultados teóricos e simulados de $\eta_{2} / \eta_{1}$; (A) bomba 35; e (B) bomba 36.

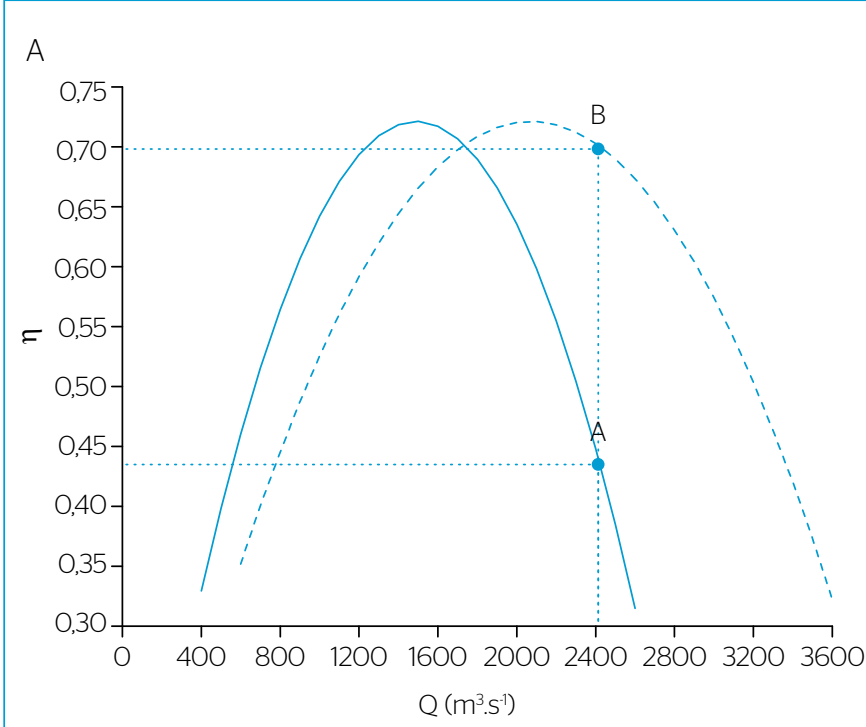

$\mathrm{N}=1,00$

\section{B}

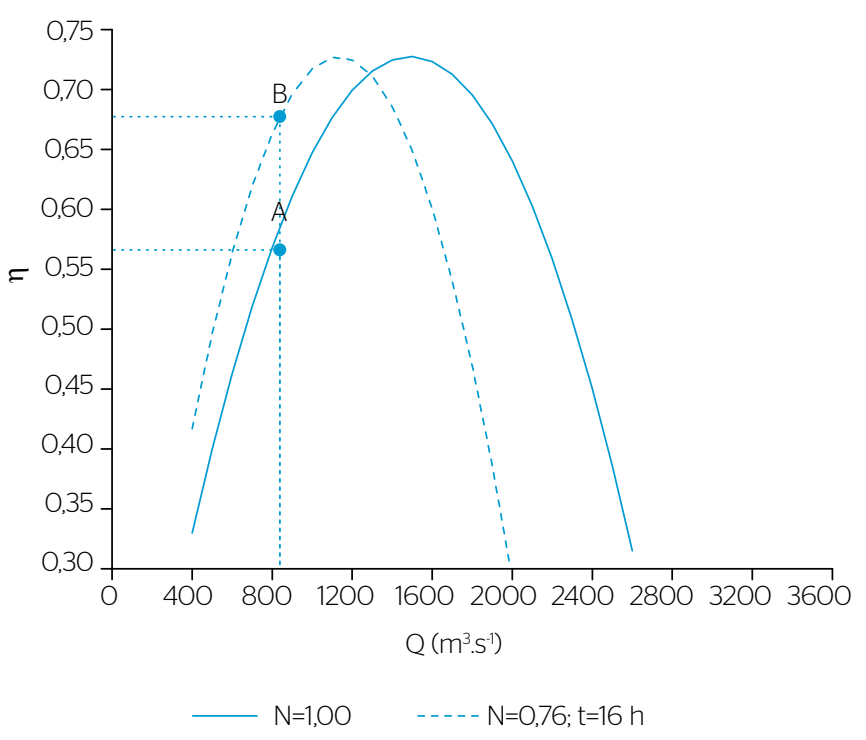

Figura 11 - Curvas teóricas $\eta \times Q$ e eficiências previstas pelo EPANET 2, versões original (ponto A) e modificada (ponto B); (A) bomba 36 em $t=4 h$; e (B) bomba 36 em $t=16 \mathrm{~h}$. 


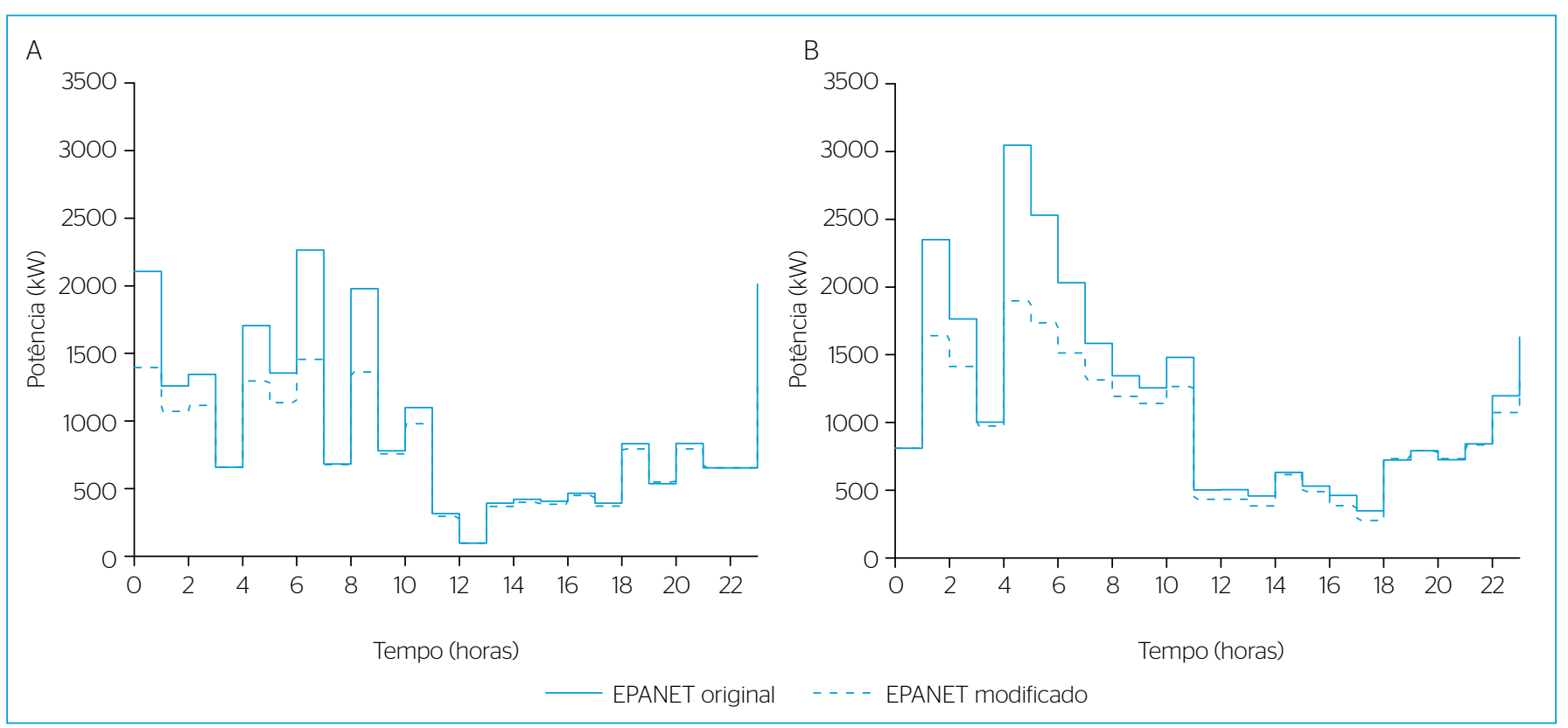

Figura 12 - Potências estimadas pelo EPANET 2, versões original e modificada; (A) bomba 35; e (B) bomba 36.

Tabela 1 - Potências estimadas pelo EPANET 2, versões original e modificada.

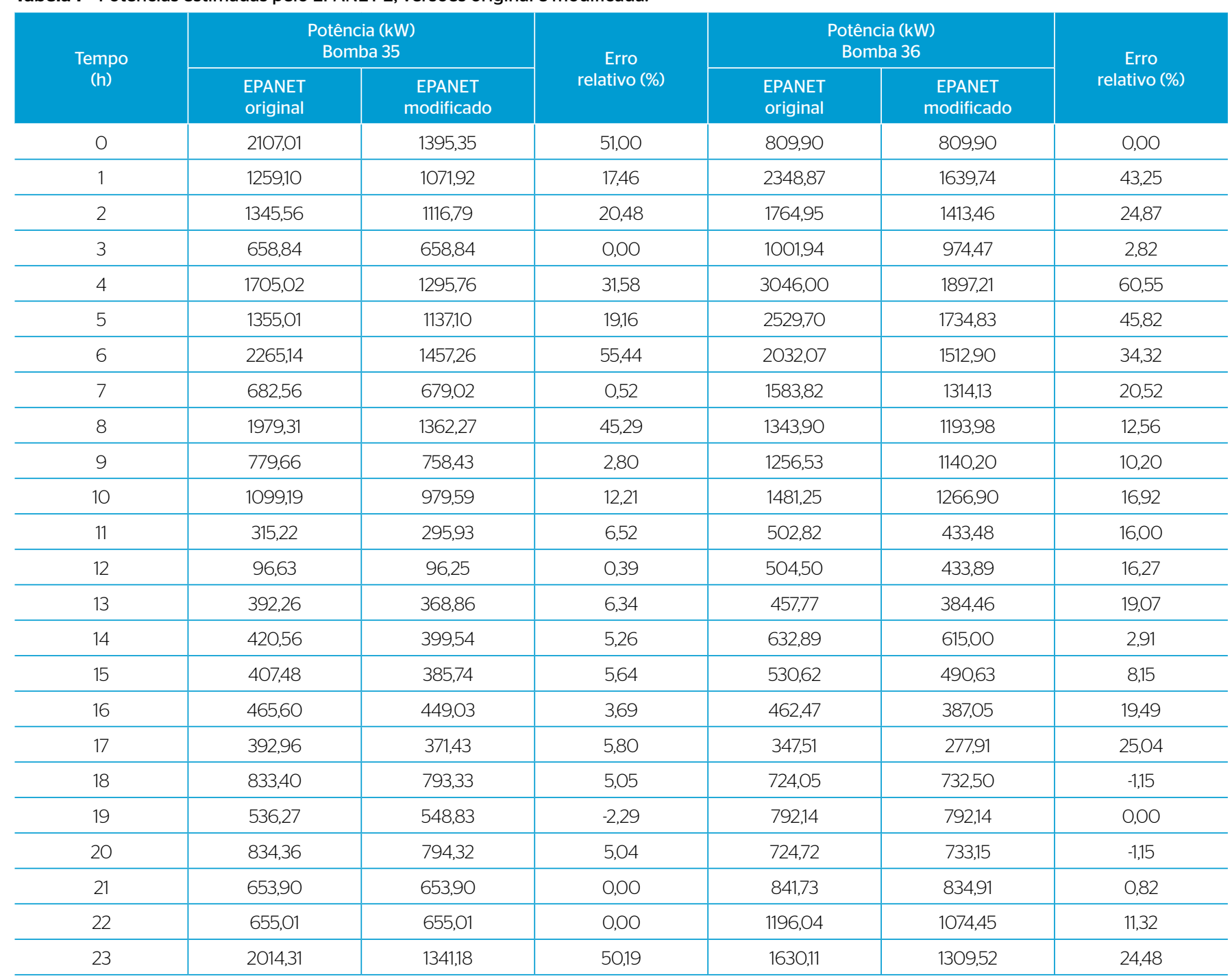


$0,82 \%(\mathrm{t}=21 \mathrm{~h})$, para a bomba 36 , sendo registradas nos intervalos de tempo correspondentes às potências mínimas das bombas. Já o maior erro entre as potências estimadas foi de $55,44 \%(\mathrm{t}=6 \mathrm{~h})$, para a bomba 35 , e de $60,55 \%(t=4 \mathrm{~h})$ para a bomba 36 , sendo registradas nos intervalos de tempo correspondentes às potências máximas das bombas.

A Tabela 2 apresenta as informações quanto ao consumo de energia contidas no relatório gerado pelo EPANET 2, versões original e modificada, ao final das simulações dinâmicas realizadas com a rede Anytown, para condições de bombeamento com eficiência variável. As informações relacionadas ao custo da energia do bombeamento foram omitidas por esse não ter sido contemplado nesta pesquisa. Nota-se que as bombas permaneceram em funcionamento durante todo o ciclo operacional. Para ambas as bombas, a eficiência média prevista pelo EPANET 2 original foi inferior à prevista pelo EPANET 2 modificado, o que pode ser explicado pelo fato daquele ter subestimado a eficiência das bombas durante a maior parte do ciclo operacional. Como os demais parâmetros são inversamente proporcionais à eficiência, os valores foram superestimados pelo EPANET 2 original. O erro relativo entre as eficiências médias estimadas pelo EPANET 2, versões original e modificada, foi igual a $10,74 \%$ para a bomba 35 , e igual a $13,02 \%$ para a bomba 36 . Quanto às energias consumidas por unidade de vazão $\left(\mathrm{kWh} . \mathrm{m}^{-3}\right)$ previstas, o erro relativo foi igual a $16,18 \%$, para a bomba 35 , e igual a $18,64 \%$ para a bomba 36 . Em relação às potências médias estimadas, o erro relativo foi igual a $21,97 \%$ para a bomba 35 e igual a $22,02 \%$ para a bomba 36 . No tocante às potências máximas previstas, o erro relativo foi igual a $55,44 \%$ para a bomba 35 e igual a $60,56 \%$ para a bomba 36 .

\section{CONCLUSÕES}

Neste trabalho, uma rotina baseada na fórmula de Sárbu e Borza (1998) é implementada no código-fonte do EPANET 2 para a correção do cálculo da eficiência de bombas de rotação variável. A influência dessa intervenção nos resultados previstos pelo

Tabela 2 - Dados de consumo de energia para condições de bombeamento com eficiência variável.

\begin{tabular}{l|c|c|c|c}
\multirow{2}{*}{ Indicador } & \multicolumn{2}{|c|}{ EPANET 2 original } & \multicolumn{2}{c}{ EPANET 2 modificado } \\
\cline { 2 - 5 } & $\begin{array}{c}\text { Bomba } \\
35\end{array}$ & $\begin{array}{c}\text { Bomba } \\
36\end{array}$ & $\begin{array}{c}\text { Bomba } \\
35\end{array}$ & $\begin{array}{c}\text { Bomba } \\
36\end{array}$ \\
\hline Utilização (\%) & 100,00 & 100,00 & 100,00 & 100,00 \\
\hline Eficiência média (\%) & 61,51 & 60,35 & 68,91 & 69,38 \\
\hline Consumo (kWh/m³) & 0,79 & 0,70 & 0,68 & 0,59 \\
\hline Potência média $(\mathrm{kW})$ & 969,02 & 1189,36 & 794,45 & 974,69 \\
\hline Potência máxima $(\mathrm{kW})$ & 2265,14 & 3046,10 & 1457,26 & 1897,21 \\
\hline
\end{tabular}

programa é avaliada em simulações dinâmicas, em período estendido, realizadas com uma rede hipotética de referência. A rotina é acionada somente se informada, como dado de entrada do modelo, a curva eficiência versus vazão das bombas na velocidade de rotação nominal. Nas simulações com bombeamento sob eficiência variável, os resultados de eficiência previstos pela versão modificada do simulador hidráulico se mostraram coerentes com os valores teóricos esperados. Demonstrou-se, portanto, que a rotina foi acionada apenas nas simulações em que as bombas operavam sob eficiência variável e que as intervenções realizadas no código-fonte não afetaram o funcionamento do algoritmo de análise hidráulica original para bombeamentos sob eficiência constante.

O EPANET 2 original ou subestimou ou superestimou a eficiência das bombas, com predomínio dos valores subestimados. Os maiores erros relativos de eficiência foram registrados para a condição de aumento na velocidade de rotação das bombas. Como a eficiência é inversamente proporcional à potência, essa foi superestimada pelo simulador hidráulico original na maior parte do ciclo operacional, chegando a alcançar erros relativos superiores a 50\% em ambas as bombas. O simulador hidráulico EPANET 2 original não leva em conta a mudança na curva eficiência versus vazão da bomba quando essa tem sua velocidade de rotação alterada e, desse modo, prevê valores incorretos para eficiência, potência e consumo de energia de bombas de rotação variável.

O consumo de energia do sistema previsto pela versão original do EPANET 2 foi $22 \%$ maior do que o previsto pela versão modificada. Tal discrepância aponta que o uso do EPANET 2 como ferramenta de apoio à avaliação econômica de projetos de implantação e/ou de intervenção em sistemas de transmissão de água com BRV pode superestimar o consumo de energia, com reflexos nos custos do projeto. Por outro lado, a versão modificada do EPANET 2 apresentada neste trabalho constitui uma alternativa mais apropriada para a avaliação preliminar de projetos dessa natureza, já que representa de forma mais acurada o comportamento de bombas de rotação variável.

Recomenda-se a investigação dos limites de aplicação da fórmula de Sárbu e Borza (1998) para BRV, considerando aspectos como o intervalo de variação da velocidade de rotação e características geométricas da bomba. Sugere-se ainda a aplicação da versão modificada do EPANET 2 em estudos comparativos de otimização da operação de sistemas de distribuição de água equipados com BRV.

\section{AGRADECIMENTOS}

Os autores agradecem ao Conselho Nacional de Desenvolvimento Científico e Tecnológico (CNPq) pelo apoio financeiro. 


\section{REFERÊNCIAS}

CARLSON, S. \& WALBURGER, A. (2007) Energy index development for benchmarking water and wastewater utilities. Denver: AWWA Research Foundation.

COUTINHO, R.S. (2015) Simulação computacional de bombas com velocidade de rotação variável no EPANET. Dissertação (Mestrado em Engenharia do Meio Ambiente) - Universidade Federal de Goiás, Goiânia.

GIUSTOLISI, O.; LAUCELLI, D.; BERARDI, L. (2013) Operational optimization: water losses versus energy costs. Journal of Hydraulic Engineering, v. 139, n. 4, p. 410-423.

GULICH, J.F. (2003) Effect of Reynolds number and surface roughness on the efficiency of centrifugal pumps. Journal of Fluids Engineering, v. 125, n. 4, p. 670-679.

IBARRA, D. \& ARNAL, J. (2014) Parallel programming techniques applied to water pump scheduling problems. Journal of Water Resources Planning and Management, v. 140, n. 7, 06014002.

KUREK, W. \& OSTFELD, A. (2014) Multiobjective water distribution systems control of pumping cost, water quality, and storage-reliability constraints. Journal of Water Resources Planning and Management, v. 140, n. 2, p. 184-193.

MARCHI, A. \& SIMPSON, A.R. (2013) Correction of the EPANET inaccuracy in computing the efficiency of variable speed pumps. Journal of Water Resources Planning and Management, v. 139, n. 4, p. 456-459.

MORTON, W.R. (1975) Economics of AC adjustable speed drives on pumps. IEEE Transactions on Industry Applications, v. 3, n. IA-11, p. $282-286$

OSTERWALDER, J. (1978) Efficiency scale-up for hydraulic turbo-machines with due consideration of surface roughness. Journal of Hydraulic Research, v. 16, n. 1, p. 55-76.
ROSSMAN, L.A. (2000a) EPANET 2 users manual. Cincinnati: USEPA.

ROSSMAN, L.A. (2000b) EPANET programmer's Toolkit Manual. Water supply and water resources division, national risk management research laboratory. Cincinnati: USEPA.

SÁ MARQUES, J.A.A. \& SOUSA, J.J.O. (2007) Hidráulica urbana: sistema de abastecimento de água. 1 reed. Coimbra: Imprensa da Universidade de Coimbra.

SÁRBU, I. \& BORZA, I. (1998) Energetic optimization of water pumping in distribution systems. Mechanical Engineering, v. 42, n. 2. p. 141-152.

SIMPSON, A.R. \& MARCHI, A. (2O13) Evaluating the approximation of the affinity laws and improving the efficiency estimate for variable speed pumps. Journal of Hydraulic Engineering, v. 139, n. 12, p. 1314-1317.

TODINI, E. \& PILATI, S. (1988) A gradient algorithm for the analysis of pipe networks. In: COULBECK, B. \& CHOUN-HOU, O. (Eds.) Computer applications in water supply: vol. 1 - systems analysis and simulation. Taunton: Research Studies Press. p. 1-20.

WALSKI, T.M.; BRILL JUNIOR, E.D.; GESSLER, J.; GOULTER, I.C.; JEPPSON, R.M.; LANSEY, K.; LEE, H.L (1987) Battle of the network models: epilogue. Journal of Water Resources Planning and Management, v. 113, n. 2, p. 191-203.

WOOD, D.J. \& REDDY, L.S. (1995) Using variable speed pumps to reduce leakage and improve performance. In: CABRERA, E. \& VELA, A.F. (Eds.) Improving efficiency and reliability in water distribution systems. Netherlands: Springer. p. 135-163.

WU, W.; SIMPSON, A.; MAIER, H.; MARCHI, A. (2O12) Incorporation of variable-speed pumping in multiobjective genetic algorithm optimization of the design of water transmission systems. Journal of Water Resources Planning and Management, v. 138, n. 5, p. 543-552. 